\title{
Endocrine disrupting metals lead to alteration in the gonadal hormone levels in Nigerian e-waste workers
}

\author{
Osaretin Godwin Igharo*,**, John I. Anetor**, Oladele Osibanjo***, \\ Humphrey Benedo Osadolor*, Emmanuel C. Odazie*, and Zedech Chukwuemelie Uche**
}

ABSTRACT
BACKGROUND
Electronic waste (e-waste) is known to contain thousands of toxic chemicals
and metals, many of which have identified endocrine disruption potentials
even at low blood concentrations resulting from occupational and
environmental exposures. E-waste crude reprocessing in Nigeria is massive
and a growing number of Nigerians are occupationally exposed. The present
study aimed to determine changes in gonadal hormone levels associated
with occupational crude e-waste reprocessing in Nigerian male e-waste
workers.

\section{METHODS}

This cross-sectional study which was carried out in Benin City, SouthSouth Nigeria recruited male e-waste workers $(n=63)$; and apparently healthy non e-waste workers $(n=41)$, as exposed and unexposed participants respectively. Male fertility hormones which includes luteinizing hormone (LH), follicle stimulating hormone (FSH), testosterone (TESTO) and prolactin (PROL); as well as progesterone (PROG), estrogen (EST) and inhibin (INH) were determined using enzyme linked immunosorbent assay (ELISA) methods; whole blood levels of chromium, cadmium, arsenic and mercury were determined using Inductively Coupled Plasma Mass Spectrometry.

\section{RESULTS}

Levels of TESTO, PROG, LH and FSH; as well as PROL and EST were significantly lower in e-waste workers compared with unexposed participants $(p<0.05)$. In contrast, serum INH was significantly higher in ewaste exposed population compared with the unexposed. In addition, chromium correlated significantly and negatively with testosterone, progesterone and estrogen, while inhibin correlated positively with chromium. Similar correlation patterns were observed for cadmium, arsenic and mercury.

\section{CONCLUSION}

Nigerian e-waste workers experienced reduced levels of key gonadal hormones and this may be associated with occupational exposure to endocrine disrupting metals in e-waste.

Keywords: Endocrine disruptors, e-wastes, toxic metals, male reproductive hormones
*Department of Medical Laboratory Sciences, School of Basic Medical Sciences, College of Medical Sciences, University of Benin, Benin City, Nigeria **Department of Chemical Pathology, Toxicology and Micronutrient Metabolism Unit, College of Medicine, University of Ibadan, Ibadan, Nigeria *** Department of Chemistry and Basel Convention Coordinating Centre for Training and Technology Transfer for the Africa Region, University of Ibadan, Oyo State, Nigeria

\section{Correspondence:}

Osaretin Godwin Igharo

Department of Medical Laboratory Sciences, School of Basic Medical Sciences, College of Medical Sciences, University of Benin, Benin City, Nigeria Email: osaretin.igharo@uniben.edu Phone: +2348038664896

Date of first submission, January 15, 2018

Date of final revised submission, April 18,2018

Date of acceptance, April 19, 2018

This open access article is distributed under a Creative Commons AttributionNon Commercial-Share Alike 4.0 International License

Cite this article as: Igharo OG, Anetor JI, Osibanjo O, et al. Endocrine disrupting metals lead to alteration in the gonadal hormone levels in Nigerian ewaste workers. Univ Med 2018;37:6574. doi: 10.18051/UnivMed.2018.v37. $65-74$ 


\section{INTRODUCTION}

Electrical and electronic waste (e-waste) which is also known as waste electrical and electronic equipment (WEEE) can be referred to as any end-of-life equipment that is dependent on electrical currents or electromagnetic fields in order to function properly. This end-of-life equipment include small and large household appliances; information technology and telecommunications equipment; lighting equipment; electrical and electronic tools, toys, and leisure and sports equipment; medical devices; monitoring and control instruments; and automatic dispensers. Components of electrical and electronic equipment such as batteries, circuit boards, plastic casings, cathode-ray tubes, activated glass, and lead capacitors are also grouped as e-waste. ${ }^{(1)}$ E-waste also includes used electronics which are destined for reuse, resale, salvage, recycling, or disposal. ${ }^{(2)}$ It is estimated that $20-50$ million tons of e-waste are produced annually worldwide in which the United States, Western Europe, China, Japan, and Australia are the major producers. ${ }^{(3,4)}$ Nigeria is currently undergoing a rapid advancement in information and communication technology (ICT). A very significant proportion of ICT users in Nigeria rely on second-hand equipment from developed countries, primarily from Europe and North America. The management of e-waste in Nigeria is a serious environmental and health issue that requires serious consideration. It was reported that Nigeria is the major e-waste dumping ground in Africa. ${ }^{(5)}$

E-waste products contain intricate blends of plastics and chemicals, which when not properly handled can be harmful to humans and pose environmental hazard. ${ }^{(6)}$ E-waste contains more than 1000 different chemical substances which fall under 'hazardous' and 'non-hazardous' categories. The presence of toxic elements like lead, mercury, arsenic, cadmium, selenium and hexavalent chromium and flame retardants in ewaste classifies them as hazardous waste. Many environmental chemicals of concern both globally and across Africa are categorized as endocrinedisrupting chemicals (EDCs). ${ }^{(7)}$ Occupational exposure to heavy metals predisposes workers to metal toxicity. ${ }^{(7)}$ The mechanism of toxicity of some heavy metals still remains unknown, although enzymatic inhibition, impaired antioxidants metabolism, and oxidative stress may play a role. Heavy metals generate many of their adverse health effects through the formation of free radicals, resulting in deoxyribonucleic acid (DNA) damage, lipid peroxidation, and depletion of protein sulfhydryl (for example, glutathione). ${ }^{\left({ }^{8}\right)}$

A substantial number of environmental pollutants such as heavy metals (arsenic, lead, cadmium, mercury), polychlorinated biphenyls, dioxin, polycyclic aromatic hydrocarbons, phthalates, bisphenol A, pesticides and alkylphenols have been shown to cause endocrine disruption and are thus referred to as endocrine disruptors. ${ }^{(9)}$ These endocrine disruptors can cause adverse effects in organisms or their progeny following the disruption of endocrine systems. ${ }^{(10)}$ The endocrine system is involved in normal growth and sexual development. The endocrine system is comprised of key organs such as the hypothalamus, the pituitary gland, the pineal gland, the thyroid gland, the parathyroids, the thymus gland, the pancreas, the adrenal glands, the ovaries, and the testes. These glands secrete substances known as hormones, which are chemical messengers released into the bloodstream, where they travel to other organs and stimulate these or their receptors. ${ }^{(11)}$ There are three general classes of hormones which are proteins and polypeptides, steroids and amino acidderived hormones. ${ }^{(12)}$ Proteins and polypeptides include hormones secreted by the anterior and posterior pituitary glands, the pancreas (insulin and glucagon), the parathyroid gland (parathyroid hormone), and many others. Steroids are secreted by the adrenal cortex (cortisol and aldosterone), the ovaries (estrogen and progesterone), the testes (testosterone), and the placenta (estrogen and progesterone). Derivatives of the amino acid tyrosine, are secreted by the thyroid (thyroxine 
and triiodothyronine) and the adrenal medullae (epinephrine and norepinephrine. ${ }^{(12,13)}$

Previous studies have shown changes in the concentration of hormones in workers occupationally exposed to heavy metals such as lead $(\mathrm{Pb})$. These changes were proposed to be mediated at the hypothalamic-pituitary level. The reproductive toxicity of lead was documented by the Greeks and Romans centuries ago and is known to cause reproductive and developmental toxicity at different levels in an organism. ${ }^{(14)}$ Lead toxicity in the male reproductive system is long known despite the controversies on the effect of low to moderate doses or route and duration of exposure involved. ${ }^{(15)}$ Human evidence suggests that lead may cause reduced semen quality without any effect on endocrine function as determined by studies of men occupationally exposed to lead ${ }^{(16)}$ Cadmium (Cd), an extremely toxic element has also been reported to have deleterious effects on the reproductive process, causing retardation of growth, sterility and embryotoxic effects. It was demonstrated that the harmful effects of cadmium are associated with interaction with other toxic metals such as lead and environmental pollutants such as fossils and petroleum by-products. ${ }^{(17-19)}$ Testicular changes due to cadmium toxicity have been seen in a variety of animal models at different stages of growth and maturity. Cadmium-induced testicular pathogenicity includes severe haemorrhage, oedema, necrosis and atrophy, as well as reduction in counts and motility of sperm and decreases in the testosterone concentrations in plasma and testes. ${ }^{(15)}$ The daily pattern release of plasma testosterone and luteinizing hormone (LH) levels was also found to be affected by cadmium. In addition, plasma levels of pituitary hormones, for example, $\mathrm{LH}$, follicle stimulating hormone (FSH), prolactin (PROL) and adrenocorticotropic hormone (ACTH), were also modified after cadmium exposure. ${ }^{(15)}$

Arsenic-induced male reproductive toxicity has also been documented. Other studies have reported that arsenic caused decrease in plasma levels of LH and FSH. ${ }^{(20)}$
It was also reported that chromium (VI) is present in e-waste and that chromium (VI) compounds are more toxic than chromium (III). (21) It was reported that workers exposed to chromium (VI) had significantly higher serum FSH concentration and lower sperm count and motility, lower seminal plasma zinc levels, lactate dehydrogenase (LDH), and LDH-C4 ${ }^{(22)}$, and significantly higher percentage of abnormal sperm than control workers, but other semen quality parameters did not differ between them. ${ }^{(32)} \mathrm{A}$ further report showed that blood chromium positively correlated with the percentage of abnormal sperm in the exposed workers. ${ }^{(22)}$

In 2014, Nigeria generated about 219 kilotons of e-waste, which is largely recycled by the informal sector. ${ }^{(24)}$ Most studies on e-waste in Nigeria were mainly on environmental and animal studies. There has been paucity of human population based studies.

The present study aimed to determine changes in gonadal hormone levels associated with occupational crude e-waste reprocessing in Nigerian male e-waste workers.

\section{METHODS}

\section{Research design}

The study was designed as a cross-sectional study with purposive approach in the recruitment of participants into the study groups. The groups comprised of e-waste occupationally exposed participants (e-waste workers) and nonoccupationally and minimally environmentally exposed; apparently healthy participants (unexposed or control participants). The study was carried out from June 2014 to June 2016. Other aspects of this extensive study were concluded in 2017.

\section{Study Area}

This study was carried out in the metropolitan city of Benin which is the capital of Edo State located in South-South Nigeria. It is a city approximately 25 miles north of the Benin River and situated 200 miles by road east 
of Lagos. It has an estimated population of $1,147,188$ according to the 2006 census .

\section{Study sample}

The study sample consisted of male workers $(n=63)$ occupationally exposed to e-waste in Benin City with duration of 5 years and above. The control subjects $(n=41)$ were recruited from apparently healthy non-exposed male subjects in Ugbowo Campus community, Benin City. The subjects in the exposed group were adult male workers who were above 18 years old with no serious health challenge in recent years and had been engaged in the work for at least 5 years and are currently doing the work. The five years duration of exposure is based on the E-waste Risk Assessment Report of Adaramodu and colleagues. ${ }^{(6)}$ The control subjects were recruited from the Ugbowo community, had not been exposed to e-waste and were apparently healthy without medical complication in the past 5 years. The control subjects were males aged 18 years and above. Individuals who were below 18 years of age, engaged in the e-waste work for less than 5 years, apparently unhealthy or with serious health challenge in recent years and have worked for 5 years but currently not engaged in the work, were excluded from the exposed group. Individuals who were below 18 years of age, exposed to e-waste, unhealthy or on therapy were excluded from the control group.

\section{E-waste workers}

Male Waste Electric and Electronic Equipment (WEEE) workers who were daily involved with four main work tasks, namely, purchasing/reception (i.e., buying and/or receiving second-hand electrical and electronic equipment (EEE)); dismantling (i.e., all work tasks involving manual dismantling of the goods); repair (i.e., all work tasks involving repair, like circuit board cleaning, soldering, rewiring, etc.) and resale (reselling repaired or inadvertently functional WEEE/EEE, or components thereof), formed the group of e-waste workers enrolled into the study.

\section{Unexposed participants (Controls)}

Non-occupationally and minimally environmentally exposed, and apparently healthy age-and sex-matched participants formed the unexposed or control group. These included fortyone (41) participants recruited from the Ugbowo Campus Community of the University of Benin, Benin City, Edo State, Nigeria.

\section{Ethical Approval}

The protocol for this pilot study was approved by the Health Research Ethics Committee of the University of Ibadan/University College Hospital, Ibadan, Nigeria (UI/UCH EC Registration Number: NHREC/05/01/2008a). Subjects for this study were adults who were adequately briefed on the research protocol and informed consent was obtained prior to sample collection.

\section{Sample collection}

Approximately 5 milliliters of venous blood was collected from test subjects (e-waste workers) and control subjects using standard phlebotomy techniques. Blood samples obtained were dispensed into plain (anticoagulant-free) specimen bottles to obtain serum after clotting and centrifugation at 3000 revolutions per minute for 3 minutes. Analysis of samples for the generation of research data was carried out using the well-preserved and labeled samples.

\section{Laboratory analysis}

Hormonal assays were done with ELISA and the results were read with the Awareness Technology product STAT FAX ${ }^{\circledR}$ Model 4700 microstrip reader, serial number: 4700-1333. (Awareness Technology Inc., Palm City, FL 34990, USA.). The INH assay was done by immunoassay method using Beckman Coulter ELISA kit. Immunoassays of LH, TESTO, PROL, PROG, and EST were carried using Accubind ELISA Microwells kits produced by Monobind Inc., Lake Forest, CA 92630, USA. Whole blood levels of chromium, cadmium, arsenic and mercury were determined using 
Table 1. Sociodemographic characteristics of the respondents by study groups

\begin{tabular}{lccc}
\hline \multirow{2}{*}{ Variables } & \multicolumn{2}{c}{ Study participants } & \multirow{2}{*}{ p-value } \\
\cline { 2 - 3 } & $\begin{array}{c}\text { E-waste workers } \\
\mathbf{n = 6 3 ( \% )}\end{array}$ & $\begin{array}{c}\text { Controls } \\
\mathbf{n = 4 1}(\mathbf{\%})\end{array}$ & $<0.001^{*}$ \\
\hline Age group (years) & $42(66.7)$ & $4(9.8)$ & \\
$20-29$ & $19(30.2)$ & $34(82.9)$ & $3(7.3)$ \\
$30-39$ & $2(3.2)$ & $29 \pm 1.1$ & 0.548 \\
$40-49$ & $31 \pm 2.0$ & $12(29.3)$ & \\
Mean age & $22(34.9)$ & $29(70.7)$ & 0.482 \\
Smoking status & $41(65.1)$ & $11(26.8)$ & \\
Ever smoked & & $30(73.2)$ & \\
Never smoked & $21(33.3)$ & & \\
Alcohol use & $42(66.7)$ & & \\
Ever used & & & \\
Never used & & & \\
\hline
\end{tabular}

* Significant at $\mathrm{p}<0.05$

Inductively Coupled Plasma Mass Spectrometry based on the method of Fong et al. ${ }^{(25)}$

\section{Statistical analysis}

Statistical analyses including descriptive statistics were carried out using the Statistical Package for Social Scientists (SPSS) version 16.0. All values were to be expressed as mean \pm standard deviation. The independent t-test was used to determine significant differences in test parameters in the test and controls groups. Differences were considered significant when $\mathrm{p}<0.05$.

\section{RESULTS}

The mean age of the e-waste workers is $31.0 \pm 2.0$ years while that of the non-exposed respondents is $29.0 \pm 2.0$ years. Two-thirds of the e-waste workers are within the age range of 20-29 years, while most controls (non-e-waste workers) are within the age range of 30-39 years. For smoking status of the respondents, 'never smoked' was high for both the exposed and non-exposed study groups. For alcohol use, 'never used' was high for both the exposed and non-exposed respondents.

Levels of male fertility hormones in exposed and unexposed participants are shown in Table 2. Correlation between heavy blood metals and male fertility hormones are shown in Table 3. Levels of TESTO, PROG, LH and FSH; as well as PROL and EST were significantly lower in e-waste workers compared with unexposed participants $(\mathrm{p}<0.05)$. In contrast, serum INH was significantly higher in e-waste exposed population compared with the unexposed. In addition, a significant negative correlation of chromium with TESTO, PROG and EST was observed ( $\mathrm{p}=0.006,0.042$ and 0.000 respectively). Inhibin positively correlated with chromium $(\mathrm{p}=0.001)$. Similar correlation

Table 2. Levels of male fertility hormones in exposed and unexposed participants

\begin{tabular}{lccc}
\hline Male fertility hormone & Exposed $(\mathbf{n}=\mathbf{6 3})$ & Unexposed $(\mathbf{n}=\mathbf{4 1})$ & p value \\
\hline LH (IU/L) & $2.62 \pm 0.25$ & $3.66 \pm 0.29$ & 0.009 \\
FSH (IU/L) & $3.61 \pm 0.28$ & $4.73 \pm 0.31$ & 0.011 \\
TESTO (ng/mL) & $1.56 \pm 0.14$ & $3.09 \pm 0.18$ & 0.000 \\
PROL (ng/mL) & $3.14 \pm 0.48$ & $4.87 \pm 0.66$ & 0.035 \\
PROG (ng/mL) & $0.09 \pm 0.02$ & $0.18 \pm 0.03$ & 0.008 \\
EST (pmol/L) & $47.25 \pm 4.63$ & $133.84 \pm 9.35$ & 0.000 \\
INH (ng/L) & $18.89 \pm 1.85$ & $6.94 \pm 1.39$ & 0.008 \\
LH (IU/L) & $2.62 \pm 0.25$ & $3.66 \pm 0.29$ & 0.009 \\
\hline
\end{tabular}

LH luteinizing hormone; FSH: follicle stimulating hormone; TESTO: testosterone : PROL: prolactin; PROG: Progesterone; EST: estrogen; INH: inhibin 
Table 3. Correlation between heavy metals and male fertility hormones among participants

\begin{tabular}{cccccccl}
\hline Heavy metals & LH & FSH & TESTO & PROL & PROG & EST & INH \\
\hline $\mathrm{Pb}$ & $-0.018 \AA$ & -0.018 & 0.049 & -0.017 & -0.090 & -0.163 & 0.063 \\
$\mathrm{Cr}$ & -0.053 & 0.005 & $-0.304^{* *}$ & 0.161 & $-0.226^{*}$ & $-0.456^{* *}$ & $0.367^{* *}$ \\
$\mathrm{Cd}$ & 0.367 & -0.040 & $-0.439^{* *}$ & 0.144 & $-0.305^{* *}$ & $-0.523^{* *}$ & $0.432^{* *}$ \\
$\mathrm{As}$ & -0.155 & -0.145 & $-0.299^{* *}$ & -0.028 & -0.145 & $-0.255^{*}$ & 0.189 \\
$\mathrm{Hg}$ & 0.189 & 0.038 & $-0.470^{* *}$ & -0.093 & -0.201 & $-0.459^{*}$ & 0.189 \\
\hline
\end{tabular}

*Significant $(p<0.05)$; **Highly significant $(p<0.001)$. ${ }^{\circledR}$ : coefficient of correlation $(r)$; Note: Pb: plumbum; Cr: chromium; CD: cadmium; As: arsenic; Hg: mercury; LH: luteinizing hormone; FSH: follivcle stimulating hormone; TESTO: testosterone; Prol: prolactin; PROG: Progesterone; EST: estrogen; INH: inhibin

patterns were observed for cadmium, arsenic and mercury.

\section{DISCUSSION}

The present study demonstrates that demographic and lifestyle indicators in the form of age, alcohol and tobacco use were comparable in the exposed and control groups, ruling out their confounding influences on the data obtained. Additionally, the observed significantly lower levels of TESTO, PROG, LH and FSH; as well as PROL and EST in e-waste workers compared with unexposed participants may be associated with endocrine disrupting effects of known ewaste-borne chemicals / metals.

This observation may further be fortified by the observed significant negative correlation of chromium with TESTO, PROG and EST in the e-waste exposed population.

Electronic waste (e-waste) is known to contain thousands of toxic chemicals and metals, many of which have identified endocrine disruption potentials even at low blood concentrations resulting from occupational and environmental exposures. ${ }^{(1-3)}$.

It is well known that exposure to toxic levels of metals causes pathological conditions to living organisms. ${ }^{(5)}$ In human, metals may cause damage to different body organs and systems. ${ }^{(9)}$ According to Pizent et al., ${ }^{(21)}$ metals may affect the male reproductive system directly, when they target specific reproductive organs, or indirectly, when they act on the neuroendocrine system. These effects can be long lasting and irreversible if Sertoli cells are disrupted during foetal development. The number of Sertoli cells determines the number of sperm produced in adulthood, because each Sertoli cell can support only a finite number of germ cells that develop into sperm. Sertoli cells proliferate during the foetal, neonatal and pre-pubertal period, and each of these periods is particularly sensitive to the adverse effects of metals. ${ }^{(6)}$

Whole blood heavy metals concentration of the subjects studied in this work has previously been reported ${ }^{(25)}$ In the study, it was observed that heavy metals in the exposed participants were significantly elevated. Accumulation of metals in the epididymis, prostate, vesicular seminalis or seminal fluid may impair progressive sperm motility especially by causing the formation of varicocele. ${ }^{(21)}$ In addition, metals can cause hormonal imbalance by affecting the neuroendocrine system, disrupting the secretion of androgens from Leydig cells or inhibin B from Sertoli cells. ${ }^{(26)}$ The disruption of spermatogenesis in men at any stage of cell differentiation can decrease the total sperm count, increase the abnormal sperm count, impair the stability of sperm chromatin or damage sperm DNA. There is growing evidence that oxidative stress is implicated in the pathogenesis of male infertility. ${ }^{(27)}$

As observed in this study, the differences in the male fertility hormones analyzed between the exposed and unexposed participants may indicate the influence of toxic metals on the reproductive system of the exposed participants. For example, LH and FSH are anterior pituitary hormones, in males; LH stimulates the interstitial (Leydig) cells in the testes to produce testosterone while FSH stimulates the Sertoli cells of the testes to produce 
inhibin which provides the main direct negative feedback control of FSH biosynthesis and release from the anterior pituitary, it also has little effect on LH release. A moderate exposure to lead has been reported to be associated with changes in the male endocrine functions, particularly affecting the hypothalamic-pituitary axis. Since the release of these hormones is controlled by the hypothalamic-pituitary axis, the decrease observed in the levels of LH, FSH, TESTO, PROL, PROG and EST may therefore be attributed to the effect of lead and other metals with similar chemical reactivity on the hypothalamic-pituitary axis of the occupationally exposed subjects. Inhibin which is produced by the Sertoli cells of the testes in response to FSH stimulation also provides the main direct negative feedback control of FSH biosynthesis and release from the anterior pituitary. ${ }^{(12)}$ This may further reduce the level of FSH in the exposed subjects. The repression of synthesis and release of $\mathrm{LH}$ and FSH have the secondary effect of repressing the release of remaining hormones down-stream the hormonal regulatory pathway. Further, LH and FSH also show negative correlations with lead, however, these correlations were statistically insignificant even at confidence limit of $95 \%$. Testosterone, secreted by the Leydig cells located in the interstitium of the testis, is essential for growth and division of the testicular germinal cells, which is the first stage in the formation of sperm cells. FSH stimulates the Sertoli cells; without this stimulation, the conversions of the spermatids to sperm cells (the process of spermiogenesis) will not occur. ${ }^{(12)}$

Similarly, oestrogens, formed from testosterone by the Sertoli cells when they are stimulated by FSH, are probably also essential for spermiogenesis. ${ }^{(12)}$ Oestrogen was drastically lowered in the exposed participants as compared to the mean concentration in the unexposed participants. These three key hormones controlling spermatogenesis were significantly lowered in the exposed studied group, the decrease in the hormones controlling spermatogenesis in this group may result in low sperm count and infertility.
Endocrine disruptors are natural products or synthetic chemicals that interfere with the synthesis, secretion, transport, binding, action or elimination of natural hormones that are responsible for the maintenance of homeostasis, development, reproduction and behaviour. Dose, duration and timing of exposure at critical periods of life are important considerations for assessing the adverse effects of endocrine disruptors. Several reports suggest that many chemicals released into the environment can affect normal endocrine function. ${ }^{(28)}$ Heavy metals are known endocrine disruptors, for example, arsenic, lead, boron, mercury, cadmium, antimony, aluminum, cobalt, chromium, lithium) have been found to exert adverse effects on the reproductive axis of human and experimental animals. Men working in battery plants and exposed to toxic levels of lead demonstrated adverse effects on their reproductive capacity. ${ }^{(6)}$ Plasma levels of pituitary hormones, for example, LH, FSH, PROL and adrenocorticotropic hormone (ACTH), were also modified after cadmium exposure. ${ }^{(15)}$ The endocrine disrupting properties of metals may be the sole cause of the differences in the hormonal concentrations between the exposed and unexposed participants, this is also supported by the correlation of the male fertility hormones and toxic metals (Table 3). As observed in these tables, most of the male fertility hormones correlated negatively with toxic metals, only inhibin correlated positively with all metals analysed. Correlations of $\mathrm{Cr}, \mathrm{Cd}$ and $\mathrm{Hg}$ with inhibin were highly statistically significant while others were not. This increase in inhibin concentration caused by toxic metals may impair hormonal function as inhibin has negative feedback effect on FSH and LH as earlier stated.

Strong negative correlations were observed between TESTO and $\mathrm{Cr}, \mathrm{Cd}$, As and $\mathrm{Hg}$, these correlations were statistically significant (Table 3 ). It was reported that workers exposed to chromium (VI) had significantly higher serum FSH concentration and lower sperm count and motility, lower seminal plasma zinc levels, lactate dehydrogenase (LDH), and LDH-C4. Cadmium- 
induced testicular pathogenicity includes severe haemorrhage, oedema, necrosis and atrophy, as well as reduction in counts and motility of sperm and decreases in the testosterone concentrations in plasma and testes. The daily pattern of release of plasma TESTO and LH levels were also found to be affected by cadmium. ${ }^{(15)}$ Arsenic caused decrease in plasma levels of LH and FSH. Paternal exposure to mercury in humans does not appear to cause infertility or malformations, but a study of pregnancy outcome among the wives of men occupationally exposed to $\mathrm{Hg}$ showed an increased incidence of spontaneous abortion. ${ }^{(29)}$

Additionally, EST showed strong negative correlations with $\mathrm{Cr}, \mathrm{Cd}$, As and $\mathrm{Hg}(\mathrm{P}=0.000$, $0.000,0.021$ and 0.000 respectively). EST is also essential for spermiogenesis. ${ }^{(13)}$ The strong negativity observed in the correlation of TESTO and EST with toxic metals shows the effect of the toxic metals on the endocrine functions of the e-waste workers. The workers are therefore at risk of developing pathological conditions that are associated with reproductive endocrine dysfunction, the effect of which may cumulatively lead to sterility and cancer.

Metal toxicity exacerbates oxidative stress which causes different pathological processes including lipid peroxidation, DNA oxidation and enzyme inactivation. Peroxidation of lipids leads to the formation of reactive products including acrolein, malondialdehyde (MDA) and 4hydroxyl-2-nonenal as major products. ${ }^{(30)} \mathrm{MDA}$ concentration was also found to increase in liver and kidney after cadmium exposure. ${ }^{(30)}$ These lipid peroxidation products have the ability to bind to virtually all kinds of biomolecules. When they bind to DNA, the binding may result in obstructed and abnormal replication during cell division, especially in actively dividing cells during spermatogenesis. DNA damage in testis may accelerate the process of germ cell apoptosis, also known as programmed cell death. ${ }^{(50)}$ This can lead to a decline in sperm counts resulting in infertility. Several studies have indicated a significant increase in the levels of apoptotic spermatozoa in the semen of infertile men. ${ }^{(51,52)}$ The oxidative damage to mitochondrial DNA (mtDNA) is also known to occur in all aerobic cells that are rich in mitochondria, including spermatozoa. Multiple mtDNA deletions in spermatozoa could arise through a free radical-driven event occurring at the spermatogonial cell stage and can account for reproductive failure in some men. ${ }^{(31)}$

Also, the binding of lipid peroxidation products to tissue proteins may lead to autoimmune disease. Modification of self antigens in the course of oxidative stress, by adduct formation with reactive products of lipid peroxidation, 4-hydroxy-2-nonenal (HNE) being one of the most commonly involved, is generally regarded to as a mechanism by which concomitant modification of self and neoantigen formation may lead to the breaking of tolerance to self antigens and, thus, to the pathogenesis of autoimmune disease. ${ }^{(54)}$ Abnormally high levels of HNE-protein adducts have been detected in the sera of children affected by autoimmune diseases. ${ }^{(32)}$ According to this view, cross-linking of HNE with self antigens would be instrumental in creating neoantigens from formerly tolerated autoantigens and, thus, initiating autoimmunity. ${ }^{(32)}$ Formation of adducts with spermatozoa structural protein may be the implicating pathogenic mechanism in the production of antisperm antibodies in women; however this has not been demonstrated. ${ }^{(32)}$ The e-waste workers studied in this work are exposed to toxicity from many metals, it can therefore be said that they are experiencing synergistic toxicity as a result of the exposure.

The limitations of this study include the fact that most e-waste workers in the study area did not meet the inclusion criteria for the study, a factor that limited us to only carry out a pilot study with a subset of the exposed population. From the outcome of this pilot study, the future directions and implications are that extensive studies on the toxicology of e-waste in the growing exposed human population in Nigeria should be undertaken. 


\section{CONCLUSION}

This study seems to demonstrate a remarkable association between e-waste occupational exposure and disruption of male fertility hormone status in the studied population.

\section{ACKNOWLEDGEMENT}

The study was financed by the Ministry of Education for support of research in NigeriaT (Tertiary Education Trust Fund)

\section{CONFLICT OF INTEREST}

None declared.

\section{CONTRIBUTORS}

This work was carried out in collaboration between all authors. IOG, AJI. and OO designed the study, wrote the protocol, and wrote the first draft of the manuscript. IOG, OHB, OEC and UZC managed the literature searches, the experimental process and data analysis. All authors read and approved the final manuscript.

\section{REFERENCES}

1. United Nations Environment Programme(UNEP). Basel convention on the control of transboundary movements of hazardous waste and their disposal. Châtelaine, Switzerland: United Nations Environment Programme; 2014.

2. Sthiannopkao S, Wong MH. Handling e-waste in developed and developing countries: initiatives, practices, and consequences. Sci Total Environ 2013;463-464:1147-53. doi: 10.1016/j. scitotenv.2012.06.088.

3. Cobbing M. Toxic tech: not in our backyard. Uncovering the hidden flows of e-waste. Amsterdam : Greenpeace International;2008.

4. Davis G, Herat S. Opportunities and constraints for developing a sustainable e-waste management system at local government level in Australia. Waste Manag Res 2010;28:705-13. doi: 10.1177/0734242X09343008..

5. Terada C. Recycling electronic wastes in Nigeria: putting environmental and human rights at risk.
Northwestern J. Intl Human Rights 2012;10:15472.

6. Adaramodu AA, Osuntogun AO, Ehi-Eromosele CO. Heavy metal concentration of surface dust present in e-waste components: the Westminister electronic market, Lagos case study. Res Environ 2012;2:9-13. doi: 10.5923/j.re.20120202.02

7. Bergman A, Heindel JJ, Jobling S, et al. State of the science of endocrine disrupting chemicals. Geneva, Switzerland: United Nations Environment Programme and the World Health Organization; 2012.

8. Morcillo P, Esteban MA, Cuesta A. Heavy metals produce toxicity, oxidative stress and apoptosis in the marine teleost fish SAF-1 cell line. Chemosphere 2016;144:225-33. DOI: https:// doi.org/10.1016/j.chemosphere.2015.08.020.

9. Balabanic DABE, Rupnik MC, Klemencic AKD. Negative impact of endocrine-disrupting compounds on human reproductive health. Reprod Fertil Develop 2011; 23: 413-6.

10. Sugawara T. Screening systems for endocrine disruptors. Reproductive and Developmental Toxicology. $1^{\text {st }}$ edition. London: Academic Press of Esevier;2011.

11. Petit W, Ademec C. The endocrine system. The Encyclopedia of Endocrine Diseases and Disorders. $1^{\text {st }}$ Edition. New York: Facts on File, Inc.;2005.

12. Guyton AC, Hall JE. Textbook of Medical Physiology. $12^{\text {th }}$ edition. Elsevier Saunders Inc. Philadephia; 2010.

13. Hamadouche NA, Nesrine S, Abdelkeder A. Lead toxicity and the hypothalamic-pituitary-testicular axis. Not Sci Biol 2013;5:1-6.

14. Sadeghniat K, Aminian O, Chavoshi F, et al. Relationship between blood lead level and male reproductive hormones in male lead exposed workers of a battery factory: a cross-sectional study. Iran J Reprod Med 2013;11:673-6.

15. Swaran JS, Flora VP, Geetu S. Reproduction and developmental toxicology. $1^{\text {st }}$ ed. London: Academic Press of Elsevier;2011.

16. Pant N, Kumar G, Upadyay AD, et al. Correlation between lead and cadmium concentration and semen quality. Andrologia 2014. DOI: https: doi.org/10.1111/and12342.

17. Allouche L, Hamadouche M, Touabti A. Chronic effects of low lead levels on sperm quality, gonadotropins and testosterone in albino rats. Exp Toxicol Pathol 2009;61:503-10. doi: 10.1016/ j.etp.2008.12.003.

18. Llanos MN, Ronco AM. Fetal growth restriction is related to placental levels of cadmium, lead and 
arsenic but not with antioxidant activities. Reprod Toxicol 2009;27:88-92.

19. Tian LL, Zhao YC, Wang XC, et al. Effects of gestational cadmium exposure on pregnancy outcome and development in the offspring at age 4.5 years. Biol Trace Elem Res 2009;24:25-29.

20. Jana K, Jana S, Samanta PK. Effects of chronic exposure to sodium arsenite on hypothalamopituitary-testicular activities in adult rats: possible an estrogenic mode of action. Reprod Biol Endocrinol 2006;4:1-13.

21. Pizent A, Tariba B, Zivkovic T. Reproductive toxicity of metals in men. Arh Hig Rada Toksikol 2012; 63: 35-46. doi: 10.2478/10004-1254-63-20122151.

22. Marouani N, Tebourbi O, Mahjoub S, et al. Effects of hexavalent chromium on reproductive functions of male adult rats. Reprod Biol 2012;12: 119-33.

23. Kumar S, Sathwara NG, Gautam AK, et al. Semen quality of industrial workers occupationally exposed to chromium. J Occup Health 2005;47:424430.

24. Ogungbuyi $\mathrm{O}$, Nnorom IC, Osibanjo O, et al. Ewaste country assessment Nigeria. Secretariate of Basel Convention; Geneva, Switzerland: 2012.

25. Fong BM, Lee TS, Tam S. Determination of mercury in whole blood and urine by inductively coupled plasma mass spectrometry. J Anal Toxicol 2007;31:281-7.
26. Igharo GO, Anetor JI, Osibanjo OO, et al. Toxic metals in Nigerian electronic waste workers indicates occupational metal toxicity associated with crude electronic waste management practices. Biokemistri 2014;26:107-133.

27. Jensen TK, Bonde JP, Joffe M. The influence of occupational exposure on male reproductive function. Occup Med 2006;56:544-53.

28. Li X, Gao Y, Wang J, et al. Exposure to environmental endocrine disruptors and human health. J Public Health Emerg 2017;1:8. doi: 10.21037/jphe.2016.12.09.

29. Bashir MR, Suresh S. Developmental and reproductive disorders: role of endocrine disruptors in testicular toxicity. Reproductive and developmental toxicology. Elsevier. London. 2011: pp.904.

30. Ayala A, Munoz MF, Arguelles S. Lipid peroxidation: production, metabolism, and signaling mechanisms of malondialdehyde and 4-hydroxy-2-nonenal. Oxidative Med Cell Longevity 2014; Article ID 360438, 31 pages. DOI: http://dx.doi.org/10.1155/2014/360438.

31. Aprioku JS. Pharmacology of free radicals and the impact of reactive oxygen species on the testis. J Reprod Infertil 2013;14:158-72.

32. Pizzimenti S, Ciamporcero, Daga M, et al Interaction of aldehydes derived from lipid peroxidation and membrane proteins. Front Physiol 2013;4:242. doi: 10.3389/fphys.2013.00242 